\title{
Public Information: From Nosy Neighbors to Cultural Evolution
}

\author{
Étienne Danchin, ${ }^{1}$ Luc-Alain Giraldeau, ${ }^{2}$ Thomas J. Valone, ${ }^{3}$ Richard H. Wagner ${ }^{4}$
}

Psychologists, economists, and advertising moguls have long known that human decision-making is strongly influenced by the behavior of others. A rapidly accumulating body of evidence suggests that the same is true in animals. Individuals can use information arising from cues inadvertently produced by the behavior of other individuals with similar requirements. Many of these cues provide public information about the quality of alternatives. The use of public information is taxonomically widespread and can enhance fitness. Public information can lead to cultural evolution, which we suggest may then affect biological evolution.

A nimals often face decisions such as where to forage, with whom to mate, or where to breed, each with different fitness outcomes. To decide effectively, they need information about the various alternatives. Unless conditions are constant, inheriting useful information about the environment genetically may be impossible, and so the acquisition of information becomes beneficial.

There are two ways to acquire information (Fig. 1): by using trial-and-error tactics to interact with the physical environment (personal information), or by monitoring others' interactions with the environment (social information). Social information can be based on signalstraits specifically designed by selection to convey information. Alternatively, it can be based on cues provided inadvertently by individuals engaged in efficient performance of their activities (inadvertent social information, or ISI). The social cues of ISI may indicate the location of resources when other individuals with similar requirements for these resources are present [social attraction (1)] or their behavior is observed from a distance [i.e., local enhancement (2)]. ISI may also involve public information (PI) (3) about the quality of the resource when it is revealed by the performance of other individuals that share similar environmental requirements (4). Here we deal mostly with instances of PI use.

The reliability of ISI for bystanders resides in the fact that they are not intention-

${ }^{1}$ U.P.M.C. CNRS-UMR7625, Bât A-7e étage-Case 237, 7 quai Saint Bernard, 75252 Paris Cédex 05, France. E-mail: edanchin@snv.jussieu.fr ${ }^{2}$ Group de Recherche en Écologie Comportementale et Animale, Département des Sciences Biologiques, Université du Québec à Montréal, Case Postale 8888, Succursale CentreVille, Montréal, Québec H3C 3P8, Canada. E-mail: giraldeau.luc-alain@uqam.ca ${ }^{3}$ Department of Biology, St. Louis University, St. Louis, MO 63103, USA. E-mail: valone@slu.edu ${ }^{4}$ Konrad Lorenz Institute, Austrian Academy of Sciences, Savoyenstrasse 1a, A-1160 Vienna, Austria. E-mail: r.wagner@klivv.oeaw.ac.at ally produced; individuals providing them are selected to perform as well as possible, rather than to inform others. In particular, PI provides rich and reliable information about the quality of alternatives $(3,4)$. The benefits of using PI are that it reduces the costs associated with trialand-error learning and provides additional information that can lead to more accurate estimates of environmental parameters (5). The use of ISI can thus vary from situations where the bystander parasitizes the information (involving a cost to the performer), to commensalism (when the bystander's use of ISI is neutral to the performer), to mutualism (when both actors benefit from the use of ISI by the bystander).

\section{The Diversity of Public Information Use}

Foraging as public information. The idea that animals may observe others to get information about resource quality arose mostly in a foraging context $(6)$. For instance, when Norway rats (Rattus norvegicus) face unfamiliar food, they rely on ISI provided by the breath of com- panions to decide on the appropriate prey to eat (7). European starlings (Sturnus vulgaris; Fig. 2A) and red crossbills (Loxia curvirostra) exploit hidden prey and must probe repeatedly to estimate the current quality of a foraging patch. Both species observe their flockmates' probing success and use this as PI to decide when to leave a patch in search of another (8-10). Scrub jays (Aphelocoma coerulescens; Fig. 2B) observe and remember the food caches of conspecifics and pilfer them when given the opportunity (11). This use of ISI could involve PI if the pilferer also uses the information to estimate the

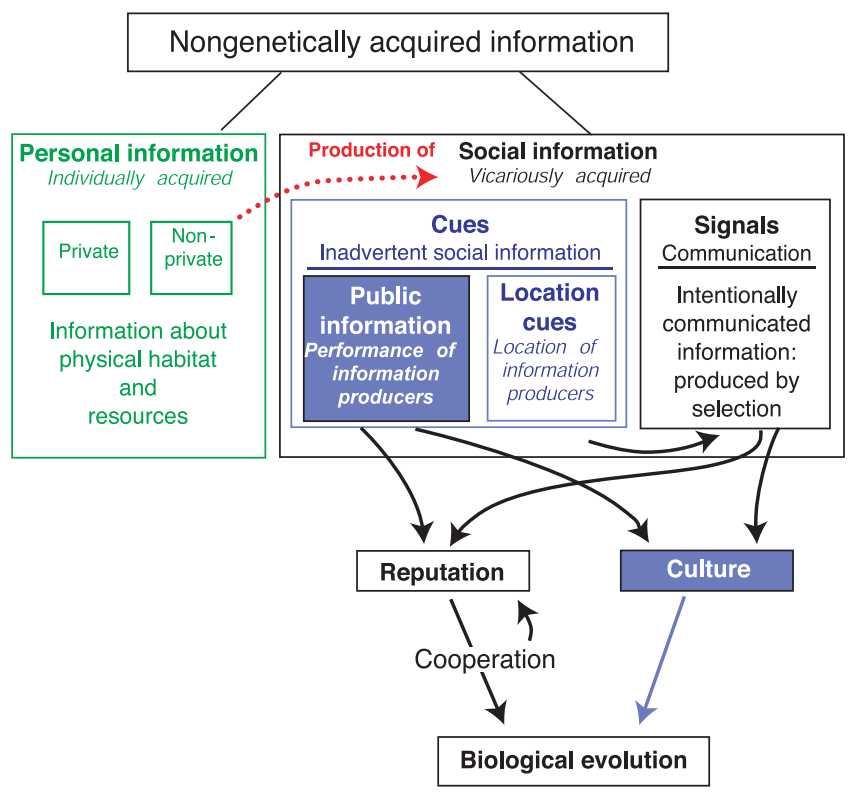

Fig. 1. The various forms of nongenetically acquired information (apart from parental effects). Information is anything that reduces uncertainty. Personal information is acquired individually by interacting with the physical environment. The interaction can generate both private information (inaccessible to others) and nonprivate information that produces social information (red arrow). A behavior can convey information by design; it is then a signal that is produced by selection. In many cases, however, social information is produced inadvertently; it is then a cue, and we refer to it as inadvertent social information (ISI). Topics covered in this review are in blue. ISI comprises cues that indicate the spatial location of resources (based on the location of the information producers) and cues produced by the performance of others, which is public information ( $\mathrm{PI})$. $\mathrm{PI}$ may play a major role in cultural transmission and evolution. The arrow from the cues box to the signals box indicates that signals directed to one party may inadvertently be used by a third party. The use of that information by others may create the selective pressures that transform ISI into signals (34). Thus, PI may be viewed in some contexts as the platform from which signals evolve. 
quality of the caches. PI can also be obtained heterospecifically; for example, nine-spined sticklebacks (Pungitius pungitius) use the feeding behavior of threespined sticklebacks (Gasterosteus aculeatus) at a poor and a rich patch to select the most profitable patch to exploit (12).

Habitat copying: Breeding success as public information. In attempting to reproduce, animals must first select an appropriate place to breed, and PI may be used in the appraisal of breeding site quality. In two lekking antelopes (Kobus kob thomasi and $K$. leche kafuensis), for example, experimentally transplanting soil from successful mating territories to less successful ones showed that females choose the mating territory according to the odors of female urine, a cue correlated with former local mating success (13). Likewise, birds such as kittiwakes (Rissa tridactyla; Fig. 2C) prospecting for a new breeding site copy the habitat choices of successful rather than unsuccessful conspecifics (14). A large-scale manipulation of the breeding performance of collared flycatchers (Ficedula albicollis) showed that local reproductive success of conspecifics was one of the cues used in the decision to disperse to other breeding areas (15). Further experimental studies of collared flycatchers have suggested that prospectors are attracted to the most successful sites as judged by parental feeding activity $(16,17)$.

The occurrence of habitat copying implies that animals prefer to settle close to successful conspecifics in order to benefit from the same favorable conditions $(4,18)$. Modeling has shown that habitat copying can lead to animal aggregation, measured
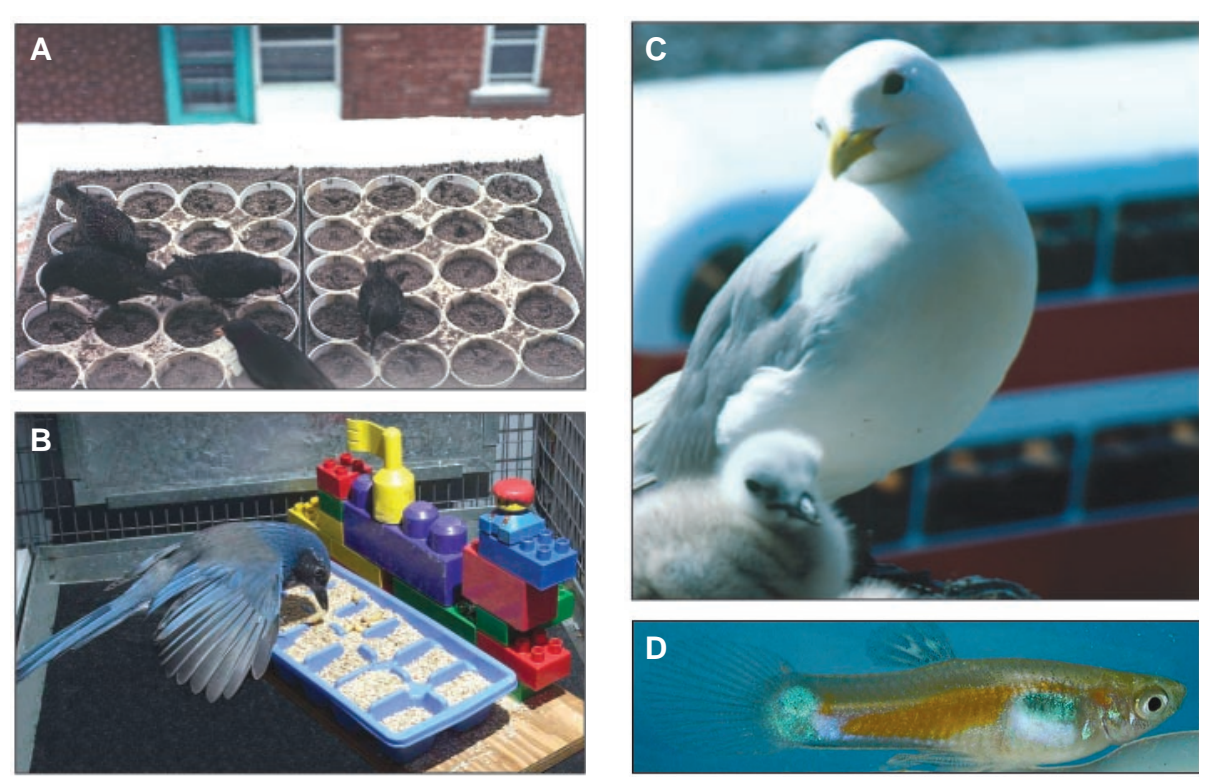

Fig. 2. Some of the animals involved in studies providing empirical evidence of the use of PI. (A) A starling (Sturnus vulgaris; photo by T. J. Valone). (B) A scrub jay (Aphelocoma coerulescens; photo by N. S. Clayton). (C) A kittiwake (Rissa tridactyla; photo by E. Danchin) and its chick. (D) A guppy (Poecilia reticulata; photo by S. Nordell).

as significant departures from a null model of dispersion (5). Once formed, these aggregations can grow because of the Allee effect (19) produced by PI use: Successful individuals provide PI about the location of high-quality habitat. In this context, colonies and other social aggregations appear as the by-product of habitat selection decisions mediated by PI $(18,20,21)$.

Mate-choice copying: Obtaining public information by watching others mate. Female mating decisions are often influenced by exposure to the mating interactions of others, such that mate-choice copying has now been reported in several species of birds and fish $(22,23)$. The mating interactions and decisions of others are a source of PI that allows an individual to assess more effectively the quality of its potential mates (22). To appraise the role of social information in mate choice, it is necessary to separate the signals deliberately produced by displaying males from the cues that are inadvertently produced by the mate choices of the copied females. Females may choose mates entirely on the basis of PI if their decisions are based on the choices of other females that reveal male attractiveness or quality. This use of PI can, in some cases, even reverse their own former choices (24). As expected, empirical data show that females use PI and hence mate-copy when choosing among potential mates of similar quality and when lacking the ability to discriminate (i.e., in situations where additional information is required to reduce uncertainty) (23, 25 ). Furthermore, mating preferences influenced by observed mating interactions could persist over time (24). Mate-choice copying was originally proposed as an explanation of the marked skew in male mating success observed in many promiscuous lekking species (26). More broadly, conspecific copying by both sexes has been suggested as a general mechanism producing skews in the distributions of the reproductive commodities being selected (21).

Eavesdropping: Watching the outcomes of others' interactions. Females can obtain information about mate quality by observing malemale interactions. Female birds, for instance, "eavesdrop" (27) on song competitions between neighboring males and then seek and obtain extra-pair fertilizations from the winning singer $(28,29)$. When dominant territorial male black-capped chickadees (Podecile atricapilla) are defeated in playback-induced countersinging contests against a simulated intruder, they lose paternity more often than do those exposed to the playback of a simulated submissive intruder (29). PI available through eavesdropping therefore plays an important role in female assessment of male quality.

Eavesdropping is also suggested by the behavior of female fighting fish (Betta splendens) that mate preferentially with winners of malemale contests $(30,31)$, whereas females that have not seen fights show indifference (32). Interestingly, the presence of a female audience increased the fighting rates of males (33), which suggests that male fights may provide both signals and cues. Eavesdropping could therefore provide a circumstance under which cues constituting PI can evolve into signals because of the audience effect (34) (Fig. 1).

Flee-response and damage as public information about danger. Acquiring information about dangers such as predators can be risky. Not surprisingly, animals have evolved the ability to detect the level of danger from the use of cues that may unavoidably accompany it. For instance, the general tendency to copy flee-responses of an entire flock (or herd) and to respond to fright or stress signs of other animals clearly involves ISI and perhaps even PI. When a predatory fish consumes a prey, the damage inflicted to the prey's cells releases chemical substances that provide PI concerning the ambient level of predation danger. These so-called alarm substances have been experimentally demonstrated in a large number of fishes (35). Even plants may use this form of PI. Wild tobacco plants (Nicotiana attenuata), for instance, apparently obtain information from airborne molecules released by damaged heterospecific neighbors. Untouched tobacco plants growing among clipped sagebrush (Artemisia tridentate) neighbors produce more flowers and seed-bearing capsules than do plants with unclipped neighbors (36), because when growing under high risk of herbivory they have a shorter life expectancy and so prematurely divert more resources to reproduction rather than growth. 
Public Information and Cultural Evolution

Cultural evolution. Many kinds of PI use are akin to true imitation (37) and thus may trigger the transmission of behavioral patterns among individuals in a process akin to culture. We define culture as the sum of traditions and information that vary among groups; the transmission of these differences across generations rests on social interactions (imprinting, imitation, learning, or teaching) that change the phenotype lastingly [definition derived from (24, $25,38)]$. Culture therefore consists of nongenetic, heritable differences among populations $(38,39)$ and requires overlapping generations that allow intergenerational transmission of phenotypic traits.

A recent review (39) underlines the striking similarities between cultural and genetic transmissions (Table 1). In particular, most of the characteristics of evolution through natural selection (variation, selective pressure, and heritability) are shared by cultural selection. A key difference, however, is that acquired cultural traits can be transmitted to the next generation [see $(37,39,40)$ for a discussion].

Examples of nonhuman culture include the song dialects of songbirds and cetaceans $(41,42)$. Song dialects are byproducts of learning processes that lead to geographic and group differences in the details of the songs used by individuals $(41,43,44)$ that may persist outside the breeding season (45). As expected of cultural evolution, dialect characteristics can change through time (46); in some cases, such as in South Pacific sperm whales (Physeter macrocephalus), dialect characteristics appear linked to foraging success (42). Moreover, in mountain whitecrowned sparrows (Zonotrichia leucophrys oriantha), males singing a local dialect have higher paternity, which suggests female preference for local dialects. The consequence is that most of the population variation at microsatellite loci that could not be attributed to individuals was attributable to differences among, rather than within, dialect regions (43). If the effect of dialects on female mating preferences is prevalent, then any two populations evolving different dialects after a sufficiently long separation may ultimately become unable to interbreed successfully (44), creating a culturally induced first step toward speciation.

Public information can trigger cultural evolution. Like true imitation (37), PI can contribute to the transmission of cultural traits $(24,39)$. Examples are numerous. Mate-choice copying implies the transmission of a phenotypic trait, a mating preference based on the behavior of predecessors, that provides information on the quality of alternative mates. Habitat copying, also based on the publicly acquired information of site quality, leads to long-lived, multigenerational traditions of site use that can generate highly skewed distributions of individuals (many in one site, few in others) that can perhaps provide a cultural explanation for the evolution of avian coloniality (18). The transmission of toolmaking and tool use $(47,48)$ usually involves the performance of the individual using the tool, and hence ISI, but perhaps involves the use of PI if the success level of the performer influences transmission. Finally, certain components of dialect transmission may involve PI if the songs of winning males are adopted more than those of losers (eavesdropping), or if song reveals something about male quality. Such possibilities have not yet been explored.

Heritability, genes, and/or culture? The widespread use of PI in animal decisionmaking requires a reappraisal of the relative contributions of culturally and genetically transmitted traits to behavioral evolution. To study this question, we must consider the definition of heritability: the fraction of phenotypic variance $\left(V_{\mathrm{P}}\right)$ that is transmitted, which is $V_{\mathrm{A}} / V_{\mathrm{P}}$, where $V_{\mathrm{A}}$ is additive genetic variance. An implicit assumption in this definition is that environmental variance $\left(V_{\mathrm{E}}\right)$ can be reduced to zero. $V_{\mathrm{E}}$ can be divided into three components:

$$
V_{\mathrm{E}}=V_{\mathrm{DE}}+V_{\mathrm{PE}}+V_{\mathrm{AC}}
$$

where $V_{\mathrm{DE}}$ is the direct effect of environmental variation, $V_{\mathrm{PE}}$ is nongenetic parental effects, and $V_{\mathrm{AC}}$ is additive cultural inheritance. Both $V_{\mathrm{AC}}$ and $V_{\mathrm{PE}}$ can lead to resemblance between parent and offspring.

In practice, however, it may be difficult to control for early parental effects. For example, some birds place various quantities of steroids and antibodies (49) in their eggs, depending on local conditions. Some studies $(50,51)$ claim that mate-choice preference is heritable, implying a genetic transmission of mating preferences. However, in most experimental designs, young are raised together, which allows for sexual imprinting. If this is so, then mate preference could be transmitted culturally rather than genetically. Part of the estimated heritability, which is usually attributed to genetic additive variance, may in fact represent parental and cultural variances $\left(V_{\mathrm{PE}}+V_{\mathrm{AC}}\right)$. Thus, what is actually measured is $\left(V_{\mathrm{A}}+V_{\mathrm{AC}}+V_{\mathrm{PE}}\right) / V_{\mathrm{P}}$. To our knowledge, no estimation of heritability has specifically controlled for the possibility of either parental or cultural transmission of preference. Variances and covariances are traditionally interpreted as pure genetic heritability (52), and even studies published in behavioral journals interpret repeatability in mate preferences as indicating genetic variation (51). Estimations of heritabilities should therefore consider the potential effect of cultural transmission, especially in studies of demonstrably acquired behavior. This would imply the rearing of young in isolation to avoid any early imprinting.

Differences between genetic and cultural evolution. The idea of a joint gene-culture effect on evolutionary processes is not new (53, 54 ). The differences between cultural and genetic transmissions of traits (Table 1 and Fig. 3) require contrasting evolutionary properties, such that evolution in a population with both genetic and cultural trait transmission differs deeply from a population without any culture (39). Cultural transmission can modify selection pressures (e.g., cultural transmission of mate preference has the potential to change sexual selection substantially). Nonrandom associations between genes and cultural traits can affect the genetic response to selection. Culture can spread rapidly, and hence can create strong selection within a population, because cultural traits can be transmitted vertically (from parent to offspring), horizontally (among members of

Table 1. Similarities and differences between genetic and cultural transmission. Much as genes are the unit of biological information (and thus of natural selection), memes are the unit of cultural information (and thus of cultural selection). Genes are stored in DNA; memes may be stored in nervous system or other tissues. Mechanisms of genetic transmission involve DNA duplication, whereas mechanisms of cultural transmission are diverse and involve imitation, imprinting, and learning, among others. Behavioral innovations are analogous to genetic mutation, and the transmission mechanisms lead to heritability in both genetic and cultural systems. Heritability of genetic traits may be lower than that of cultural traits such as language.

Genetic transmission

\begin{tabular}{|c|c|c|}
\hline & & \\
\hline Information unit (replicator) & Gene & Meme \\
\hline Information vector & DNA & Behavior and central nervous system \\
\hline Transmission mechanism & DNA duplication & $\begin{array}{l}\text { Imitation, social facilitation, } \\
\text { imprinting, learning, teaching }\end{array}$ \\
\hline Mutation & $\begin{array}{l}\text { Duplication errors; } \\
\text { pseudo-genes }\end{array}$ & Learning errors; innovation \\
\hline Impact of most mutations & Deleterious & Unknown \\
\hline Heritability & Yes (low) & Yes (moderate to high) \\
\hline $\begin{array}{l}\text { Transmission of acquired } \\
\text { characters }\end{array}$ & No & Yes \\
\hline Type of process & Darwinian & Lamarckian \\
\hline
\end{tabular}




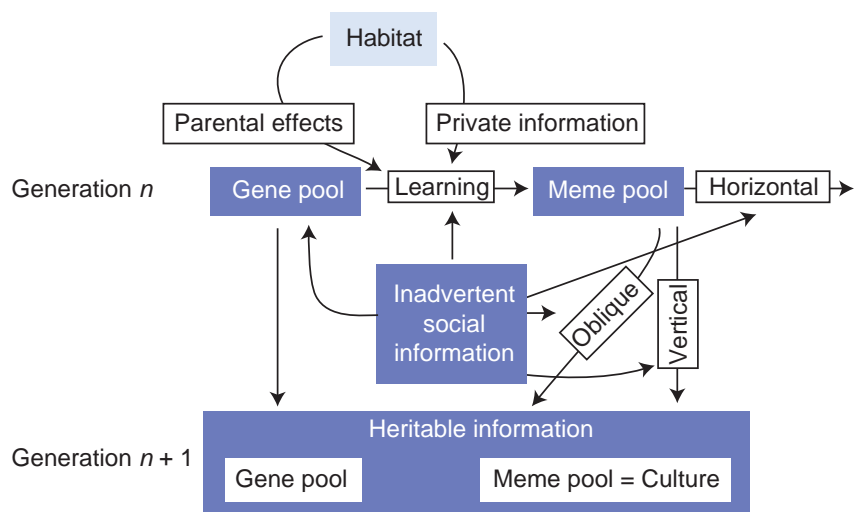

Fig. 3. The role of PI and other forms of ISI in relation to genetic and cultural transmission. During development, individuals learn environmental types through maternal effects and imitation. Learning can involve private or social information. As a major source of information extracted from conspecifics, ISI and PI in particular lead to the emergence of culture. The effects of ISI and PI as selective agents for better learning and imitation feed back to the gene pool (hence to genetic evolution). Both genetic and cultural information are then transmitted to the next generation. An important difference between genetic and cultural transmission is the latter's capacity to transmit traits both horizontally and obliquely. [Adapted from (58)]

the same generation), and obliquely (from nonparental members of the previous generation). For instance, by diminishing within-group variation and increasing among-group variation, cultural transmission and conformity to culture can enhance the effect of selection among groups relative to selection among individuals, and can account for the spread of agricultural practices $(37,39,55)$.

Public information, genes, and culture. A large number of male sexual ornaments are thought to have arisen through Fisherian sexual selection. Once female preference is fixed in a population, the evolution of Fisherian male ornaments can only move in the direction of greater exaggeration. Yet there is evidence from phylogenetic analyses that the evolution of exaggerated male traits can be reversed: Ancestral species in which females preferred bright males can give rise to daughter species in which females prefer drab males (56). This result is puzzling because it is difficult to explain how a mutant female with a genetic preference for drab males could invade a population where females prefer bright males. However, experimental results obtained with guppies (Poecilia reticulata; Fig. 2D) may provide an answer to this puzzle. Female guppies appear to have a heritable noncultural preference for brightly colored males (52). However, they can acquire a preference for drab males, provided that they observe other females mating preferentially with such males for a sufficiently long period of time (25). Mating preferences can thus be reversed culturally through the use of PI.

A Fisherian interpretation for the evolution of exaggerated male traits rests on the observation that when females mate with their preferred kind of male, their offspring inherit both the male's exaggerated trait and the female's preference (57). This joint inheritance of trait and preference was interpreted as implying that genes that code for male traits become linked to genes that code for female preferences. Genes, however, need not be invoked at all, and the proper expression should be that heritable male traits become linked to heritable female preference. For instance, males' territory acquisition ability can be influenced by the quality of their natal territory. When this is so, territory quality can be inherited without necessarily involving any genetic transmission, a process called ecological inheritance (58). Similarly, as was shown in guppies, female preference can be socially acquired when species have overlapping generations. An association can arise between an ecologically inherited male ability to acquire territories and a culturally inherited female mate preference for males with such abilities.

An association between a genetic and a culturally inherited trait is also possible. For instance, territory acquisition ability can be genetically inherited, whereas female preference for this trait may be culturally acquired. When this is assumed to be so, gene-culture models reveal the existence of a parameter equivalent to linkage disequilibrium between genes that code for learning capacities and cultural states (59), implying that links between genes and culture can be similar to links among genes themselves. The reversion of female preferences in some lineages (56) may thus be explained by cultural inheritance of mate preferences, with the cultural effect arising from the use of PI.

\section{Conclusions}

PI is a widespread phenomenon that is emerging as a potential unifying concept in fields that involve decision-making processes in which individuals can extract information from others to assess resource quality. The use of PI can enrich evolutionary models and can have marked effects on evolutionary predictions. Future research should explore the extent to which evolutionary scenarios are affected by the use of PI.

A number of important questions about PI still need to be explored. We know, for instance, that PI use is not limited to cognitively sophisticated organisms, because even plants appear able to use it. However, it is likely that an organism's mechanisms for acquiring PI and processing the information may be associated to the extent to which PI will influence its behavior.

Although information is often considered as a highly valuable commodity, it is not valuable in all circumstances. In some cases, using PI may be incompatible with gathering personal information, leading to a trade-off between the two. The potential for such a trade-off highlights the importance of ascertaining the relative value of public versus personal information.

PI may be the major driving force in social evolution, both as a selective agent for behavioral adaptations and as a population-level process that can change evolutionary dynamics, in particular through its implications in cultural evolution. Because evidence for the use of PI in decision-making comes from very different taxa, it suggests that cultural evolution may be ancestral and perhaps more widespread than is currently thought. A productive avenue of research will be to investigate whether a number of traits currently assumed to be genetically transmitted could involve cultural processes. Moreover, although much work has been devoted to exploring how biological evolution affects culture, we suggest that evolutionary biologists should also consider how cultural evolution influences biological evolution (37). A major issue will be to tease apart heritability that results from the interaction of genes, maternal effects, and culture. PI may well provide an effective tool for such studies.

In summary, the ability of individuals to use PI unites a range of topics as diverse as foraging, predation, mate choice, habitat selection, and colony formation. The study of PI, which is inadvertently produced, may also enhance our understanding of the evolution of signals and hence communication. Finally, the public information framework provides a useful approach for examining the evolution of culture and its influence on biological evolution.

\section{References and Notes}

1. J. Veen, Behaviour 20 (suppl.), 1 (1977).

2. N. J. Buckley, Am. Nat. 149, 1091 (1997).

3. T. J. Valone, Oikos 56, 357 (1989).

4. E. Danchin, D. Heg, B. Doligez, in Dispersal, J. Clobert, E. Danchin, A. A. Dhondt, J. D. Nichols, Eds. (Oxford Univ. Press, New York, 2001), pp. 243-258.

5. B. Doligez, C. Cadet, E. Danchin, T. Boulinier, Anim. Behav. 66, 973 (2003).

6. P. Ward, A. Zahavi, Ibis 115, 517 (1973).

7. B. G. Galef, Can. J. Psychol. 44, 311 (1990).

8. J. J. Templeton, L. A. Giraldeau, Behav. Ecol. 6, 65 (1995).

9. J. J. Templeton, L. A. Giraldeau, Behav. Ecol. Sociobiol. 38, 105 (1996)

10. J. W. Smith, C. W. Benkman, K. Coffey, Behav. Ecol. 10, 54 (1999).

11. N. J. Emery, N. S. Clayton, Nature 414, 443 (2001).

12. I. Coolen, Y. van Bergen, R. L. Day, K. N. Laland, Proc. R. Soc. London Ser. B 270, 2413 (2003).

13. J. C. Deutsch, R. J. C. Nefdt, Nature 356, 596 (1992).

14. E. Danchin, T. Boulinier, M. Massot, Ecology 79, 2415 (1998). 
15. B. Doligez, E. Danchin, J. Clobert, Science 297, 1168 (2002).

16. T. Pärt, B. Doligez, Proc. R. Soc. London Ser. B 270 1809 (2003)

17. B. Doligez, T. Pärt, E. Danchin, J. Clobert, L. Gustafsson, J. Anim. Ecol. 73, 75 (2004)

18. E. Danchin, R. H. Wagner, Trends Ecol. Evol. 12, 342 (1997).

19. F. Courchamp, T. Clutton-Brock, B. Grenfell, Trends Ecol. Evol. 14, 405 (1999).

20. R. H. Wagner, E. Danchin, T. Boulinier, F. Helfenstein, Behav. Ecol. 11, 572 (2000)

21. R. H. Wagner, E. Danchin, Anim. Behav. 65, 405 (2003).

22. S. E. Nordell, T. J. Valone, Ecol. Lett. 1, 74 (1998).

23. T. J. Valone, J. J. Templeton, Philos. Trans. R. Soc London Ser. B 357, 1549 (2002)

24. K. Witte, B. Noltemeier, Behav. Ecol. Sociobiol. 52 194 (2002).

25. L. A. Dugatkin, The Imitation Factor: Evolution Beyond the Gene (Free Press, New York, 1999).

26. W. J. Wade, S. G. Pruett-Jones, Proc. Natl. Acad. Sci. U.S.A. 87, 5749 (1990).

27. P. K. McGregor, T. M. Peake, Acta Ethol. 2, 71 (2000)

28. K. Otter et al., Proc. R. Soc. London Ser. B 266, 1305 (1999).

29. D. J. Mennill, L. M. Ratcliffe, P. T. Boag, Science 296 873 (2002).

30. R. A. Johnstone, Proc. Natl. Acad. Sci. U.S.A. 98, 9177 (2001).
31. R. F. Oliveira, M. Lopes, L. A. Carneiro, A. V. M. Canario, Nature 409, 475 (2001).

32. C. Doutrelant, P. K. McGregor, Behaviour 137, 1655 (2000).

33. C. Doutrelant, P. K. McGregor, R. F. Oliveira, Behav Ecol. 12, 283 (2001).

34. A. Lotem, R. H. Wagner, S. Balshine-Earn, Behav. Ecol. 10, 209 (1999).

35. D. P. Chivers, R. J. F. Smith, Ecoscience 5, 338 (1998).

36. R. Karban, J. Maron, Ecology 83, 1209 (2002).

37. S. Blackmore, The Meme Machine (Oxford Univ. Press, Oxford, 1999).

38. J. Soltis, R. Boyd, P. J. Richerson, Curr. Anthropol. 36, 473 (1995).

39. A. Mesoudi, A. Whiten, K. N. Laland, Evolution 58, 1 (2004).

40. E. Jablonka, M. J. Lamb, E. Avital, Trends Ecol. Evol. 13, 206 (1998)

41. D. A. Nelson, P. Marler, A. Palleroni, Anim. Behav. 50, 83 (1995)

42. H. Whitehead, L. Rendell, J. Anim. Ecol. 73, 190 (2004).

43. E. A. MacDougall-Shackleton, S. A. MacDougallShackleton, Evolution 55, 2568 (2001).

44. B. R. Grant, P. R. Grant, Biol. J. Linn. Soc. 76, 545 (2002).

45. P. S. Warren, Anim. Behav. 65, 1169 (2003).

46. G. Chilton, M. R. Lein, Condor 98, 567 (1996).

47. G. L. Hunt, R. D. Gray, Proc. R. Soc. London Ser. B 270, 867 (2003).
48. G. R. Hunt, M. C. Corballis, R. D. Gray, Nature 414, 707 (2001)

49. J. Gasparini, K. D. McCoy, C. Haussy, T. Tveraa, T. Boulinier, Proc. R. Soc. London Ser. B 268, 647 (2001).

50. V. K. lyengar, H. K. Reeve, T. Eisner, Nature 419, 830 (2002).

51. G. S. Wilkinson, H. Kahler, R. H. Baker, Behav. Ecol. 9 , 525 (1998)

52. R. Brooks, J. A. Endler, Evolution 55, 1002 (2001)

53. L. L. Cavalli-Sforza, M. W. Feldman, Cultural Transmission and Evolution: A Quantitative Approach (Princeton Univ. Press, Princeton NJ, 1981).

54. R. Boyd, P. J. Richerson, in Social Learning: Psychological and Biological Perspectives, T. R. Zentall, B. G. Galef, Eds. (Erlbaum, Hillsdale, NJ, 1988), pp. 29-48.

55. M. W. Feldman, K. N. Laland, Trends Ecol. Evol. 11, 453 (1996).

56. J. J. Wiens, Trends Ecol. Evol. 16, 517 (2001)

57. M. Andersson, Sexual Selection: Monographs in Behavior and Ecology (Princeton Univ. Press, Princeton, NJ, 1994).

58. K. N. Laland, J. Odling-Smee, M. W. Feldman, Behav. Brain Sci. 23, 131 (2000).

59. M. W. Feldman, L. L. Cavalli-Sforza, Proc. Natl. Acad. Sci. U.S.A. 81, 1604 (1984)

60. We thank A. Lotem, M. van Baalen, and two anonymous referees for constructive comments on previous versions of this manuscript.

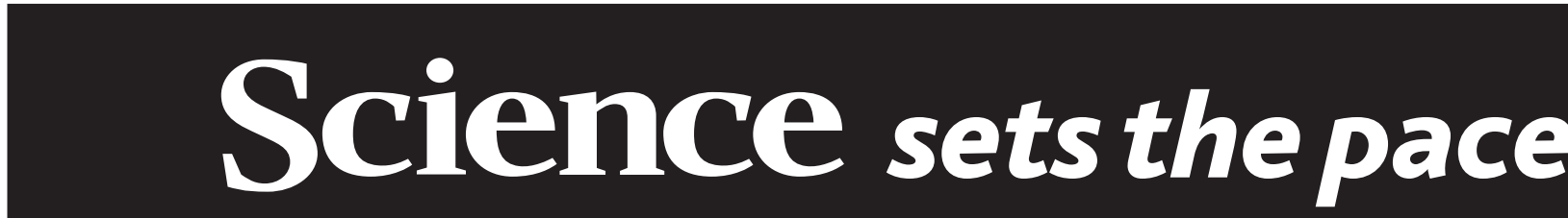

online manuscript submission

\section{www.submit2science.org}

Science can now receive and review all manuscripts electronically

online letter submission

\section{www.letter2science.org}

Have your voice be heard immediately

\section{speed submission}

